\title{
Distinct Effects of Topical Nitroglycerol on Brain Delivery of Free and Nanoparticle-Bound Drugs in Rodents
}

\author{
Anna Alekseeva ${ }^{1}$, Alexander Khalansky ${ }^{1}$, Olga Maksimenko ${ }^{2}$, Nadezhda Osipova ${ }^{2}$, \\ Kenul Abbasova ${ }^{3}$, Joerg Kreuter ${ }^{4}$, Svetlana Gelperina ${ }^{2}$ \\ ${ }^{1}$ Research Institute of Human Morphology, Cjurupy st. 3, Moscow, 117418, Russia \\ ${ }^{2}$ Drugs Technology Ltd., Rabochaya st. 2a, Khimki, Moscow region, 141400, Russia \\ ${ }^{3}$ Lomonosov Moscow State University, Faculty of Biology \\ 1-12 Leninskie Gory, Moscow, 119991, Russia \\ ${ }^{4}$ Institute of Pharmaceutical Technology, Goethe University \\ Marie-Curie-Str. 9, Frankfurt am Main, 60439, Germany \\ mariott@bk.ru; svetlana.gelperina@gmail.com
}

\section{Extended Abstract}

Introduction: Penetration of many drugs into the brain is restricted by the BBB. As shown previously, the poly(butyl cyanoacrylate) nanoparticles (PBCA NPs) coated with polysorbate 80 and poly(lactide-co-glycolide) nanoparticles (PLGA NPs) coated with poloxamer 188 could enhance brain delivery of doxorubicin and loperamide, which was evidenced by their pharmacological effects (i.e. antitumour effect against intracranial tumour and central analgesic effect, respectively) unattainable by these drugs in free form [1-3]. It is known that penetration of the low molecular and even macromolecular drugs into the brain could be improved by application of NO-donors (such as nitroglycerol). The purpose of the present study was to investigate whether nitroglycerol also could further enhance the brain delivery of nanoparticle-bound drugs.

Methods: Doxorubicin was loaded in the PBCA NPs prepared by emulsion polymerization. Wistar rats bearing the intracranial 101.8 glioblastoma received doxorubicin, either free (Dox) or bound to 250-nm PBCA nanoparticles coated with polysorbate 80 (Dox-PBCA) (i.v. $1.5 \mathrm{mg} / \mathrm{kg}$ on days 2, 5, and 8 post tumour implantation, $\mathrm{n}=20$ ). Ten animals in each group received also nitroglycerol $(1 \mathrm{mg} / \mathrm{kg})$ applied topically immediately before injection. Loperamide was loaded in 150-nm PLGA NPs prepared by a homogenization - solvent evaporation technique. The antinociceptive effect of loperamide formulations $(7 \mathrm{mg} / \mathrm{kg}$, i.v.) with and without topical nitroglycerol were evaluated in healthy rats using a tailflick test.

Results: The experiments confirmed a higher antitumor efficacy of the Dox-PBCA formulation: a $150 \%$ increase of mean survival time (IST) and 33\% of long-term survivals (>100 days), as compared to Dox (IST=65\%, no long-term survivors). Interestingly, the effect of nitroglycerol was distinct for two formulations: Dox efficacy was considerably potentiated (IST $=150 \%, 25 \%$ of long-term survivals), whereas the efficacy of Dox-PBCA was even decreased: IST $=106 \%$, one long-term survivor.

As expected, free loperamide, an opiate receptor agonist, showed only marginal antinociceptive effect in rats, whereas the nanoparticulate formulation coated with poloxamer 188 could produce a considerable central analgesia (up to $60 \%$ of the maximal possible effect). These results correlated with the previous observations. Application of nitroglycerol led to a decrease of the analgesic effect of the nanoparticle-bound loperamide - similarly to the results obtained with doxorubicin.

Conclusion: These results confirm that nitroglycerol enhances circumvention of the BBB by low-molecular Pglycoprotein substrates; however, this approach may not work for the nanoparticles in the range of 150-250 $\mathrm{nm}$.

\section{References}

[1] R. N. Alyautdin, et al., "Delivery of loperamide across the blood-brain barrier with polysorbate 80-coated polybutylcyanoacrylate nanoparticles," Pharm Res., vol. 14, pp. 325-328, 1997.

[2] S. Gelperina et al., "Drug delivery to the brain using surfactant-coated PLGA nanoparticles. Influence of the formulation parameters," Eur J Pharm Biopharm., vol. 74, pp. 157-163, 2010. 
[3] S. C. Steiniger et al., "Chemotherapy of glioblastoma in rats using doxorubicin-loaded nanoparticles," Int J Cancer., vol. 109, pp. 759-767, 2004. 